\title{
Nefropatici: dalla poesia all'Evidence Based Medicine. C'è posto per la bioetica?
}

\author{
Mario Timio ${ }^{1,2}$, Francesca Timio ${ }^{1}$ \\ ${ }^{1}$ Dipartimento di Medicina Interna e Nefrologia e Dialisi, Ospedale di Foligno, Foligno (PG) \\ ${ }^{2}$ Direzione Scientifica Centro di Bioetica, Regione Umbria, Perugia
}

\begin{abstract}
Patients with renal disease: from poetry to Evidence Based Medicine. Is there a place for bioethics?
Why some patients with renal disease start to compose poetry? They concord that this is a supplement to their clinical history and a help to physicians. They underline that poetry does not get rid of Evidencce Based Medicine (EBM) and of its scientific basis, but they find in it a support to their soul voice. It is a supplement of clinical practice and an extra kick to progress toward the hope of a better lifetime. Poetry is a help that a man offers to other men, a guide to avoid or to lessen pain and mistake. If poetry is a supplement of clinical practice, EBM has a privileged role in accompanying it when it enters in the dialysis ward. On the other hand, EBM is strictly tied up to bioethics guiding every clinical approach; then a "red thread" makes a sound choice by applying poetry to nephrology and EBM. The poetry of three renal patients explains the development of a comprehensive bioethical principle and gives us a guide to its application in everyday clinical activity. Talking about EBM and poor importance nowadays somebody gives it, an outstanding medical journal published these ironic alternatives to it: Eminence based medicine, Vehemence based medicine, Eloquence based medicine, Providence based medicine, Diffidence based medicine, Nervousness based medicine.
\end{abstract}

Keywords: Bioethics, Evidence Based Medicine, Poetry

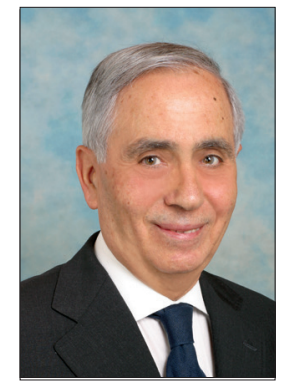

Mario Timio

Nefropatici che mettono in versi dolori e speranze

La lettura, la poesia, la prosa narrativa e l'Evidence Based Medicine (EBM), tutto può ruotare intorno alla malattia. Nella mia libreria fanno mostra di sè alcuni volumetti scritti e regalatimi da pazienti nefropatici in terapia conservativa o sostitutiva, nei quali la realtà e la fantasia incentrate sulla medicina dialogano ad armi pari. Ogni tanto ritorno alla loro lettura per trarne non solo insegnamenti di vita, ma considerazioni di bioetica.

Scrive la giovane Susanna in emodialisi da due anni: "Quante volte/mi ha consolato/questa vecchia scrivania./

Accepted: April 12, 2017

Published online: May 5, 2017

Indirizzo per la corrispondenza:

Prof. Mario Timio

Via XX Settembre 22

06121 Perugia

timma@libero.it
Ora mi chiedo se è giusto tapparsi le orecchie./Giusto o non giusto/bisogna continuare./Lacrime umane non dissetarono mai/un fiore appassito". Poche scarnite parole molto più eloquenti di dotti saggi sociologici e psicologici. Speranza invece di disperazione, accettazione invece di rabbia, amore invece di ostilità, sconfitte sopportate con composta eleganza, piccole vittorie amplificate dalla progettualità emergente. II tutto messo in versi che offrono spunti di gioia e motivi di serenità (1). Ma quanto le rime aiutano Susanna più dell'applicazione dell'EBM?

"Ascolta il canto dei grilli/e quello delle cicale/i cori degli usignoli e il suono delle campane", leggo nel libretto di Giulio, trapiantato renale, il quale si rifà a un sonetto cinese che recita: "La storia oppone il suo dolore al nostro canto ottimista/ alla nostra speranza è un monito./La nostra stella s'è scaldata per dar vita/a una specie dubbiosa che non ha mai provato il suo valore". II canto di lode alla vita, surrogato da Giulio, anche quando questa è in bilico, non è futile, poiché fornisce le coordinate di un'impalpabile realtà che diversamente sfugge. $E$ che rimane in tutta la sua validità anche quando la propria condizione da trapiantato si tramuta in dializzato. Lo si desume anche dai versi che trasudano speranza dopo il fallimento del trapianto. Anche se inconsciamente, ogni male scioglie quotidianamente un cantico di gioia alla vita e, in virtù di questo cantico, annota ancora Giulio, la vita assume 
una tinta meno buia, chiara, quasi rosea. Sì, perché si presta attenzione alle cose che rendono bella la vita o, meglio, che fanno apprezzare la bellezza della vita. Qualcuno dice che questa bellezza non la vede in dialisi, ma Giulio, come tanti altri, la scorge proprio nell'ausilio al vivere. Ma questo ausilio fallirebbe, argomentano altri scettici, se non si facesse ricorso alla Medicina Basata sulle Evidenze.

Nella poesia "Approcci in zona ospedaliera", Paola, venti anni, in emodialisi, si esprime cosi: "Disturba l'allegria/ che ammansisce il dolore/per sana rivalità./Topolino bianco/ àtono trastullo/di tale effimero potere/non confrontare/la tua opinione con la loro diagnosi". In altri versi immortala l'amore che ha appena sfiorato: "Se qualcuno/mi avesse detto/ ti amo/forse ora non starei a sognarti/a immaginare/come sarebbe il tuo amore/a ricordare/le tue mani/su di me./Sarei moglie e madre/ora son di nessuno./E mèndico/un foglio bianco". Un foglio bianco dove deporre come in una placenta accogliente la perfida malattia e la memoria, il silenzio della luce (aveva perso la vista a causa del diabete mellito) e la speranza dell'intelligenza, tutto "senza urtare i birilli" del vivere quotidiano, dove dimenticare quasi "gli occhi senza sole". Tuttavia, dopo aver afferrato con grazia, talvolta con ironia, l'arma del fare poetico, vivendo sul "filo del dolore", ma non affondandovi, poiché il timone del vascello lo regge bene con "la speranza dell'intelligenza" e, anche lei, con la fede nella medicina.

\section{Il legame tra poesia e malattia}

A questo punto qualcuno si chiede che c'entra la poesia o la narrativa con la medicina e nella fattispecie con la nefrologia o meglio con il nefropatico. Cioè con la persona che sente l'esigenza tra una dialisi e l'altra di trovare appigli per qualche certezza che cerca nell'analisi del suo divenire quotidiano e storico. E questo appiglio lo trova nella penna che stila versi che possono avere un effetto catartico o progettuale o semplicemente culturale (2). Può essere anche qualcos'altro. Come scrive Giuseppe Pontiggia la poesia o qualsiasi altro genere letterario è un aiuto che un uomo offre a un altro uomo, una guida per evitare il dolore e l'errore e porvi rimedio. Senza scriverlo, è quanto mi hanno riferito concordemente Susanna, Giulio, Paola.

Ma agli stessi ho chiesto come pongono la poesia o la prosa narrativa in rapporto alla salute loro e ai destinatari delle loro opere. La risposta è stata concorde: "un supplemento alla mia storia clinica e al lavoro del medico". Se è un supplemento vengono coinvolte la scientificità dell'approccio medico e l'EBM che la sostiene in un abbraccio che presuppone e sostanzia la bioetica. In questo apparente groviglio di scambi clinici ed epistemologici prestiamo attenzione al divenire della Medicina Basata sulle Evidenze. Chiarendo che il binomio poesia-salute designa da tempo un complesso ordine di connessioni che richiedono un serrato confronto a più voci tra la componente umanistica e scientifica. Lo sguardo sulla realtà clinica con categorie umanistiche, più che un'indebita incursione nei territori della medicina, disegna spunti discreti nell'interazione fra alcune forme alte di creazione poetica e le puntuali condizioni del disagio dialitico avvertito dai nostri pazienti-poeti.

Allora, a proposito di EBM, che può indurre a "tentazioni positivistiche", chiediamoci subito: quanto durano le "evidenze" dell'Evidence Based Medicine? (3). Chi non ricorda i magnifici volumi "Clinical Evidence" editi dal gruppo del British Medical Journal intorno agli anni 2000? Perfetti nel mettere in evidenza i migliori risultati scientifici ma anche le incertezze dei singoli studi e le relative applicazioni cliniche. Sembravano testi sacri di fatti scientifici. Sfogliateli oggi e vedrete che le notizie ivi contenute, come indicano gli stessi redattori, hanno perso la valenza clinica e sono considerate come curiosità storiche (4). Allora non in tre lustri le evidenze cliniche non sono più evidenze, ma in sei mesi, come è scritto onestamente in prefazione dei citati volumi. Perché ciò avviene? Ci viene incontro la moderna epistemologia. II fatto, nella fattispecie la realtà medica, non è sacro, contrariamente alla corrente versione classica: "Contra factum non valet argumentum". L'obiezione ce la fornisce l'epistemologo Karl Popper: nessun fatto è sacro, per la semplice ragione che ogni nostra osservazione, compresa quella medica, è imbrattata di teoria; appena parliamo di un fatto, noi gettiamo su di esso la rete linguistica dei nostri concetti e teorie e lo interpretiamo e nessuna nostra interpretazione può dirsi indiscutibile, assoluta, scritta per sempre. In altre parole, poiché la scienza, come ricorda ancora Popper, è "ipotetica, falsificabile e fallibile e la nostra conoscenza progredisce per tentativi ed eliminazioni di errori", nel fallibilismo popperiano trova la più alta espressione di razionalità l'accettazione dei limiti della scienza e, quindi, della medicina, nella convinzione del carattere temporaneo e inevitabilmente difettoso di ogni conoscenza scientifica (5). È proprio questa temporaneità alla base della breve durata delle evidenze in medicina e, quindi, della precarietà dell'EBM. Critiche e revisioni scientifiche sono alla base del continuo divenire di teorie patogenetiche e terapeutiche, propellente del procedimento metodologico per tentativi ed errori (trial and error). Secondo la teoria fallibilista di Popper applicata alla medicina e alla nefrologia, il vero problema non è quello di trovare una teoria che sia perfetta, ma di correggerne i difetti nella convinzione del suo carattere inevitabilmente precario e correggibile.

\section{Un eloquente messaggio epistemologico: "La nefrologia su palafitte"}

È proprio questa precarietà di ogni principio fisico, come di ogni teoria biologica, che offre un'immagine complessiva di "una medicina su palafitte", cioè di una medicina (o nefrologia) in continuo rimaneggiamento non solo sul piano scientifico, ma anche sull'indirizzo logistico e applicativo (6). Pensiamo alle vicende cliniche delle tre persone citate, Susanna, Giulio, 
Paola, che hanno lasciato versi di cristallina leggerezza ove vengono sublimate le difficoltà della malattia in cantici di speranza, ove tutte le sconfitte sono sopportate con composta eleganza e tutte le piccole vittorie amplificate nell'inaspettato momentaneo miglioramento e talvolta nella sopravvivenza. Tutto ciò sarebbe potuto avvenire nel cuore delle tre persone se non avessero avuto a disposizione nuovi approcci terapeutici e innovative applicazioni tecnologiche sulla base di piccoli o grandi progressi scientifici applicati alla loro malattia? Pur sapendo che le sofferenze come le gioie attivano sentimenti poetici che innescano sublimi versi poetici, aggiungo che I'iter poetico è avvenuto anche perché minuscole e talvolta insignificanti ipotesi sono diventate ciclopiche teorie sature di vantaggiose applicazioni terapeutiche che hanno sostituito o affiancato altre all'epoca vigenti. Pensiamo all'eritropoietina che ha sostituito la trasfusione di sangue o al bicarbonato al posto dell'acetato nel liquido di dialisi. Frutto della determinazione di conoscere e di sconfiggere la malattia o le sue complicanze, studiosi e ricercatori, con l'umiltà dei propri limiti e con la consapevolezza della precarietà della "nefrologia su palafitte", hanno vagliato ipotesi su ipotesi per trovare la soluzione migliore. Una soluzione di cui hanno usufruito anche i nostri tre poeti. Ed è bene che sia avvenuto così, poiché i pazienti esigono che venga loro offerta la migliore risposta alla loro domanda di cura. Lo esigono perché è la bioetica che lo impone, la quale, come ripetutamente esposto in questa rubrica, è il viatico di ogni medico verso la salute del paziente, con o senza poesia, ma sempre con la mediazione negli ultimi venti anni della Medicina Basata sull'Evidenza, con tutti i limiti sopra esposti. Completata dall'esigenza di vicinanza ai bisogni dei malati e più rispettosa del giudizio del medico o, se si vuole, più rispettosa dell'assetto epistemologico tradizionale della medicina. Un assetto che ha permesso alla medicina degli ultimi 70 anni di configurarsi più come scienza naturale e come disciplina razionale. Se l'importanza della ricerca clinica si sostituisce, come sostiene Giovanni Federspil, al valore dell'esperienza clinica individuale e del razionale fisiopatologico, l'EBM rischia di fallire nei suoi obiettivi. E ciò perché la posizione metodologica dell'EBM rappresenta in realtà il ripristino di posizioni epistemologiche sorpassate, "posizioni che si risolvono in ultima analisi in un regresso verso l'empirismo scientifico e in una sottovalutazione della razionalità della medicina" (7).

In sostanza l'EBM ha un futuro se viene considerata come una tecnica di studio in grado di far acquistare in breve tempo informazioni tecniche utili e attendibili. Al contrario, come sottolinea ancora Federspil, "se viene proposta come un vero paradigma clinico metodologico, contrassegnato da un'impostazione empirica che tende a considerare meno rilevanti le acquisizioni teoriche delle scienze di base e a ritenere invece espressione autentica della realtà medica la fenomenologia clinica, non come risulta dall'esperienza individuale del medico ma come appare da risultati epidemiologici", I’EBM può rappresentare un incompleto e scorretto assetto mentale del medico. Un assetto che può alimentare anche qualche poesia critica di epigoni tardivi di Susanna, Giulio, Paola.

\section{Alternative ironiche all'EBM}

Poesia o narrazione, di fronte alla serietà di un problema come quello dell'EBM ci sono medici che affrontano la realtà con proposte tra il serio e il faceto. E questi medici, tutt'altro che sprovveduti, veri free-lance delle soluzioni a buon mercato, riescono a pubblicare su British Medical Journal il risultato di una chiacchierata tra amici e colleghi che si chiedono che fare quando il medico si trova di fronte a un problema che non ha risposta né da indicazioni ricavate da trial né da buone evidenze cliniche.

Vengono suggerite queste alternative:

1) Eminence based medicine. È quella del collega più anziano per il quale l'esperienza è la migliore evidenza e nella quale ha una fiducia smisurata. II medico eminente è quello della pacca sulla spalla e della parola incoraggiante.

2) Vehemence based medicine. È quella del volume di voce al posto dell'evidenza. La spiegazione urlata mette K.O. il collega timido e convince gli altri della propria abilità clinica.

3) Eloquence based medicine. L'eloquenza verbale e l'eleganza sostanziale sono potenti sostituti dell'evidenza.

4) Providence based medicine. Se il medico non ha idee per affrontare il problema clinico e terapeutico, è bene lasciare la decisione nelle mani di Dio. Sfortunatamente troppi medici sono incapaci di dare alla divina provvidenza una mano nel prendere una decisione.

5) Diffidence based medicine. Alcuni medici vedono un problema e cercano di risolverlo. Altri vedono il problema e basta. Il medico diffidente non fa nulla per un senso di disperazione. Ciò ovviamente è meglio del fare qualcosa solo perché viene ferito l'orgoglio del medico per non far nulla.

6) Nervousness based medicine. In Italia si chiama Medicina Difensiva. La paura di una denuncia è un potente fattore di esagerata richiesta di esami. In questa atmosfera di paura il miglior test è quello che il medico non richiede (8).

In sintesi, tante alternative decisionali in carenza di evidenze, anche se presentate in chiave ironica. Ma chi ha mai detto che nell'ironia non ci sia un contenuto di verità che possa aiutare la scienza o l'arte medica? È un po' come nella poesia ove si indovano segmenti di verità per l'autore e il lettore.

\section{Disclosures}

Financial support: No financial support was received for this submission.

Conflict of interest: The authors have no conflict of interest. 


\section{Bibliografia}

1. Timio M. Storie di ordinaria speranza. L'ottimismo contro la malattia. Cittadella Editrice, Assisi. 1996:12.

2. Wittgenstein L. in Calimani R. I destini e le avventure dell'intellettuale ebreo. Mondadori, Milano. 1996:89.

3. Antiseri D, Timio M. La medicina basata sulle evidenze. Analisi epistemologica. Editoriale Bios. Cosenza. 2000:54.

4. AA.VV. Clinical Evidence. The International source of the best available evidence for effective health care. BMJ Publishing Group, London. 2001:xiii.
5. Popper KR. Logica della scoperta scientifica. II carattere auto correttivo della scienza. Trad. it. Einaudi, Torino. 1970:44.

6. Timio M. Il caso Di Bella e dintorni. Cittadella Editrice. 1998:33.

7. Federspil G. Premessa a: Sackett DI, Scoot Richardson W, Rosemberg W. Brian Haynes R. La medicina basata sull'evidenza. Come praticare e insegnare I'EBM. Trad. it. Centro Scientifico Editore, Torino. 1998:XVIII-XIX.

8. Isaac D, Fitzgerald D. Seven alternatives to evidence based medicine. Brit Med J. 1999;319:1618. 